\title{
Acute Stroke Care during the COVID-19 Pandemic: Reduction in the Number of Admissions of Elderly Patients and Increase in Prehospital Delays
}

\author{
Gabriel Velilla-Alonso ${ }^{a}$ Andrés García-Pastor ${ }^{b}$ Ángela Rodríguez-López ${ }^{a}$ \\ Ana Gómez-Roldós ${ }^{a}$ Antonio Sánchez-Soblechero ${ }^{a}$ Laura Amaya-Pascasio ${ }^{a}$ \\ Fernando Díaz-Otero $^{b}$ Yolanda Fernández-Bullido ${ }^{b}$ \\ Ana María Iglesias-Mohedano ${ }^{b}$ Pilar Vázquez-Alén ${ }^{b}$ Marta Vales-Montero ${ }^{b}$ \\ Antonio-Carmelo Gil-Núñez ${ }^{b}$

\begin{abstract}
aDepartment of Neurology, Hospital General Universitario Gregorio Marañón, Madrid, Spain; bDepartment of Neurology, Vascular Neurology Section - Stroke Center, Hospital General Universitario Gregorio Marañón, Madrid, Spain
\end{abstract}

\section{Keywords}

Acute stroke · Stroke care · Coronavirus · Coronavirus disease $2019 \cdot$ Spain

\begin{abstract}
Introduction: We analyzed whether the coronavirus disease 2019 (COVID-19) crisis affected acute stroke care in our center during the first 2 months of lockdown in Spain. Methods: This is a single-center, retrospective study. We collected demographic, clinical, and radiological data; time course; and treatment of patients meeting the stroke unit admission criteria from March 14 to May 14, 2020 (COVID-19 period group). Data were compared with the same period in 2019 (pre-COVID-19 period group). Results: 195 patients were analyzed; 83 in the COVID-19 period group, resulting in a $26 \%$ decline of acute strokes and transient ischemic attacks (TIAs) admitted to our center compared with the previous year ( $p=0.038)$. Ten patients $(12 \%)$ tested positive for PCR SARSCoV-2. The proportion of patients aged 65 years and over
\end{abstract}

karger@karger.com www.karger.com/ced

(c) 2021 S. Karger AG, Base

Karger ${ }^{\prime}=$ was lower in the COVID-19 period group (53 vs. $68.8 \%, p=$ $0.025)$. During the pandemic period, analyzed patients were more frequently smokers ( 27.7 vs. $10.7 \%, p=0.002$ ) and had less frequently history of prior stroke (13.3 vs. $25 \%, p=0.043$ ) or atrial fibrillation ( 9.6 vs. $25 \%, p=0.006$ ). ASPECTS score was lower (9 [7-10] vs. 10 [8-10], $p=0.032)$, NIHSS score was slightly higher (5 [2-14] vs. 4 [2-8], $p=0.122)$, onset-to-door time was higher (304 [93-760] vs. 197 [91.25-645] $\mathrm{min}, p=$ $0.104)$, and a lower proportion arrived within $4.5 \mathrm{~h}$ from onset of symptoms ( 43.4 vs. $58 \%, p=0.043$ ) during the COVID-19 period. There were no differences between proportion of patients receiving recanalization treatment (intravenous thrombolysis and/or mechanical thrombectomy) and in-hospital delays. Conclusion: We observed a reduction in the number of acute strokes and TIAs admitted during the COVID-19 period. This drop affected especially elderly patients, and despite a delay in their arrival to the emergency department, the proportion of patients treated with recanalization therapies was preserved.

(c) 2021 S. Karger AG, Basel 


\section{Introduction}

The ongoing coronavirus disease 2019 (COVID-19) pandemic has tested healthcare systems all around the world, taking some of them on the brink of collapse. Spain has been one of the European countries most affected by the pandemic. The first case of SARS-CoV-2 infection was diagnosed in Spain on January 31, 2020. On July 17, 2020 , the epidemic had left in the country a total of 260,255 confirmed cases and 28,420 deaths from the novel coronavirus [1].

In order to face the pandemic, several measures have been taken progressively at all levels, from adapted medical protocols or in-hospital clean pathways to national social distancing measures. Thus, on March 14, 2020, the Spanish government ordered a nationwide lockdown. The management of pathologies requiring urgent attention, like stroke, has been affected by all these changes [2, 3].

Our center, Hospital General Universitario Gregorio Marañón, is located in the center of Madrid, the capital of the country and of the Madrid region, the most affected of the 17 administrative regions in which it is divided Spain. Once the first wave of the pandemic has been left behind, we would like to analyze its impact on the number of acute strokes attended in our center during the first 2 months of lockdown in Spain. We also aim to analyze the differences between the demographic, clinical, and radiological characteristics of patients attended, the time course of acute stroke care, and the number of recanalization therapies performed during the COVID-19 epidemic compared with the previous year.

\section{Methods}

\section{Acute Stroke Pathway in the Madrid Region}

The Madrid region has a population of around 6.6 million inhabitants. Ten hospitals of the Madrid Regional Health Service have a stroke unit. Our stroke center provides tertiary care to a population of approximately 800,000 people. Seven of the Regional Health Service hospitals, including our center, are prepared to perform urgent mechanical thrombectomy. Each center has its own catchment population but in order to optimize resources, a thrombectomy on-call network has been organized to guarantee treatment 24/7 in the whole region. Thus, outside of working hours, there is a weekly rotating shift. Three of the 7 hospitals offer every day urgent thrombectomy for candidate patients, including those from other catchment areas [4].

\section{Study Design and Population}

This is a single-center, retrospective, observational study. Patients selected were divided in two different groups. The first group included patients meeting the stroke unit admission criteria from March 14 to May 14, 2020 (COVID-19 period group), coinciding with the first 2 months of lockdown in Spain. The second group included those admitted to the stroke unit during the same period in 2019 (from March 14 to May 14, 2019, pre-COVID-19 period group). Data were compared between both groups.

Our stroke unit admission criteria are as follows: adult patients with diagnosis of acute ischemic or hemorrhagic stroke or transient ischemic attack (TIA); arrival to the Emergency Department (ED) within $24 \mathrm{~h}$ from the onset of symptoms or last known well; and a modified Rankin Scale (mRS) score $\leq 2$ ( 3 in case of good family support). The clinical diagnosis is made by a neurologist. A basic blood test, an ECG, and a brain computed tomography (CT) scan are performed before admission to the stroke unit. In case of ischemic stroke candidate to reperfusion treatment, a CT angiography and a CT perfusion is performed.

During the COVID-19 period some measures were implemented in our center. Specific COVID-19 wards and intensive care units were created and most hospital neurologists and stroke physicians were progressively reallocated to COVID-19 wards. The stroke unit was kept as a clean area only for non-COVID-19 patients. In order to keep a clean pathway, stroke patients clinically suspicious of COVID-19 tested positive for SARS-CoV-2 PCR or those with a suggestive pulmonary infiltrate were transferred to a COVID-19 ward or a specific intensive care unit for COVID-19 patients. Patients infected with SARS-CoV-2 meeting the stroke unit admission criteria were included in the study.

Diagnose of COVID-19 was confirmed with presence of SARSCoV-2 RNA in real-time reverse transcription PCR from nasopharyngeal exudate. The molecular diagnosis was based on the 2019nCoV RT-PCR Assay Kit v1 (singleplex) by Applied Biosystems. RT-PCR was analyzed using the Applied Biosystems QuantStudio 5 or 7500 real-time PCR systems [5].

\section{Patient Data and Variables}

Demographic data, past medical history including vascular risk factors, baseline and discharge National Institutes of Health Stroke Scale (NIHSS) score, treatment with intravenous recombinant tissue-type plasminogen activator ( $r$-tPA) or thrombectomy, and final diagnosis were collected retrospectively. Alberta Stroke Program Early Computed Tomography Scale (ASPECTS) score was established by a neurorradiologist. Large vessel occlusion was defined as a CT angiography confirmed occlusion of the carotid artery, anterior cerebral artery, middle cerebral artery, posterior cerebral artery, vertebral artery, and basilar artery. Acute stroke care times analyzed included the following: onset-to-door time, defined as the period of time from onset of symptoms or last known well to ED arrival; door-to-CT time, the period from ED arrival to CT scan performance; door-to-needle time, the period from ED arrival to administration of thrombolisis; and door-to-groin time, the period from ED arrival to groin puncture for mechanical thrombectomy.

\section{Statistical Analysis}

Continuous variables are shown as medians (interquartile range) and noncontinuous variables as percentages. Differences between both groups (COVID-19 period group and pre-COVID-19 period group) were tested using $\chi^{2}$, Fisher exact test or Mann-Whitney $U$ test where appropriate. Analyses were done using SPSS version 25.0 and the level of significance was established at $p<0.05$. 
Fig. 1. Box plot of median onset-to-door time (in minutes) of the pre-COVID-19 period group and COVID-19 period group. $\mathrm{IQR}$, interquartile range.

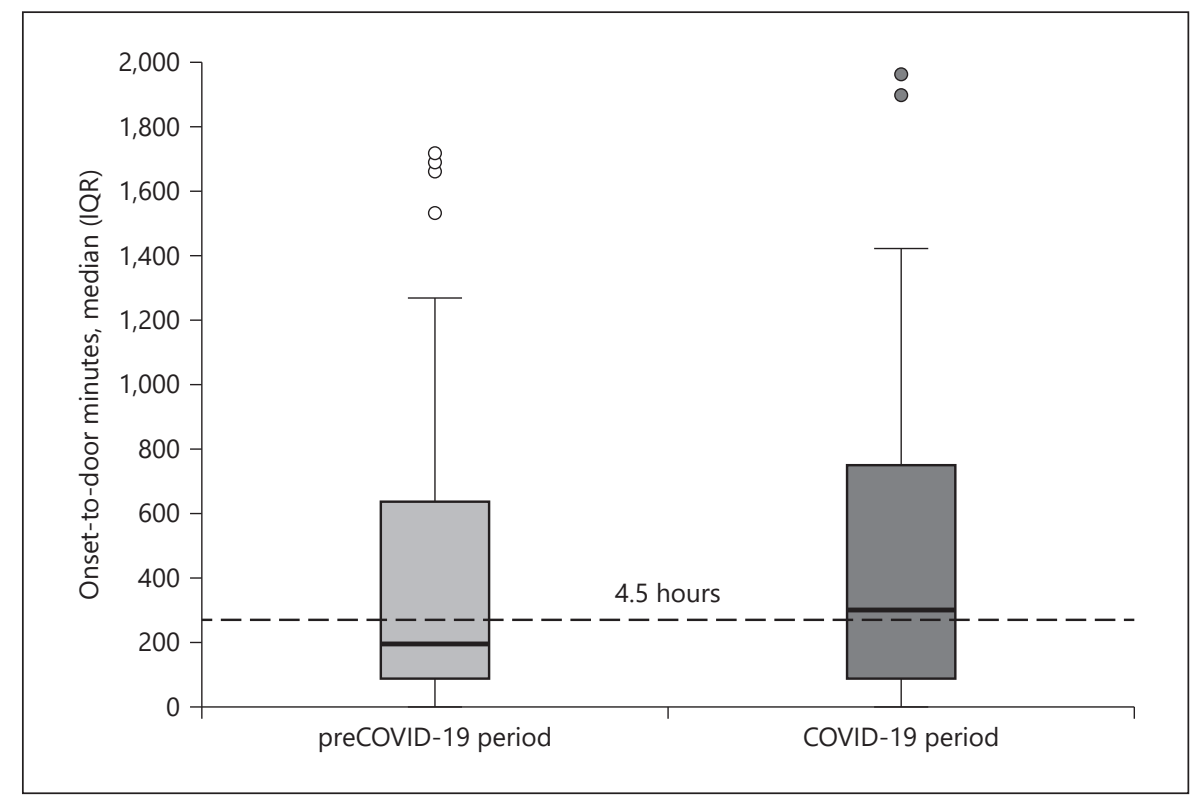

\section{Results}

From a total of 195 patients analyzed, 83 corresponded to the COVID-19 period group. This means a fall of $26 \%$ of acute strokes or TIAs attended compared with the previous year $(p=0.038)$. Ten patients $(12 \%)$ tested positive for PCR SARS-CoV-2 and did not enter the stroke unit despite meeting the admission criteria. The main baseline characteristics of patients are detailed in Table 1. The proportion of patients aged 65 years and over was lower in the COVID-19 period group (53 vs. $68.8 \%, p=0.025$ ). During the pandemic period, analyzed patients were more frequently active smokers ( 27.7 vs. $10.7 \%, p=0.002$ ) and had less frequently a prior history of stroke (13.3 vs. $25 \%, p=0.043$ ) or atrial fibrillation ( 9.6 vs. $25 \%, p=$ $0.006)$.

Stroke subtypes and ischemic stroke etiology did not differ between both groups. Within middle cerebral artery ischemic strokes $(n=77)$, ASPECTS score was lower in the COVID-19 period group (9 [7-10] vs. 10 [8-10], $p=0.032)$. We did not observe differences between the proportion of strokes of unknown onset and the frequency of large vessel occlusion. Arrival and discharge from the stroke unit NIHSS score was slightly higher in the COVID-19 period group, but there was no statistically significant difference (see Table 2).

Stroke onset-to-door time was more than $100 \mathrm{~min}$ higher during the COVID-19 period (304 [93-760] vs. 197 [91.25-645] min, $p=0.104$ ) (see Fig. 1) and a lower
Table 1. Baseline characteristics of the patients

\begin{tabular}{llll}
\hline & $\begin{array}{l}\text { Pre-COVID-19 } \\
\text { period group } \\
(n=112)\end{array}$ & $\begin{array}{l}\text { COVID-19 } \\
\text { period group } \\
(n=83)^{\mathrm{a}}\end{array}$ & $p$ value \\
\hline Demographics & & & \\
$\quad$ Median age, years (IQR) & $76(60.2-83)$ & $68(53-79)$ & 0.008 \\
Age $\geq 65$ years, $n(\%)$ & $77(68.8)$ & $44(53.0)$ & 0.025 \\
Male, $n(\%)$ & $62(55.4)$ & $43(51.8)$ & 0.623 \\
Past medical history, $n(\%)$ & & & \\
Hypertension & $88(78.6)$ & $64(77.1)$ & 0.808 \\
Diabetes & $32(28.6)$ & $20(24.1)$ & 0.485 \\
Dyslipidemia & $68(60.7)$ & $43(51.8)$ & 0.214 \\
Current smoker & $12(10.7)$ & $23(27.7)$ & 0.002 \\
Atrial fibrillation & $28(25.0)$ & $8(9.6)$ & 0.006 \\
Previous stroke/TIA & $28(25.0)$ & $11(13.3)$ & 0.043 \\
\hline
\end{tabular}

Numbers are $n(\%)$, median (IQR) as appropriate. COVID-19, coronavirus disease 2019; IQR, interquartile range; $n$, number; TIA, transient ischemic attack. ${ }^{\text {a }}$ Ten patients tested positive for PCR SARS-CoV-2.

proportion of patients arrived within $4.5 \mathrm{~h}$ from onset of symptoms ( 43.4 vs. $58 \%, p=0.043$ ). We did not find statistically significant differences between proportion of patients receiving recanalization treatment (intravenous thrombolysis or mechanical thrombectomy) or in-hospital time course, including door-to-CT time, door-to-needle time, or door-to-groin time (see Table 3). 
Table 2. Stroke characteristics

\begin{tabular}{|c|c|c|c|}
\hline & $\begin{array}{l}\text { Pre-COVID-19 } \\
\text { period group } \\
(n=112)\end{array}$ & $\begin{array}{l}\text { COVID-19 } \\
\text { period group } \\
(n=83)\end{array}$ & $p$ value \\
\hline Unknown symptom onset, $n(\%)$ & $41(36.6)$ & $26(31.3)$ & 0.443 \\
\hline Wake-up strokes, $n(\%)$ & $27(24.1)$ & $19(22.9)$ & 0.843 \\
\hline Median baseline NIHSS score (IQR) & $4(2-8)$ & $5(2-14)$ & 0.122 \\
\hline Median discharge NIHSS score (IQR) & $2(1-4.75)$ & $3(1-10)$ & 0.061 \\
\hline ASPECTS score, ${ }^{a}$ median (IQR) & $10(8-10)$ & $9(7-10)$ & 0.032 \\
\hline Large vessel occlusion, ${ }^{\mathrm{b}} n(\%)$ & $34(32.7)$ & $28(39.4)$ & 0.360 \\
\hline \multicolumn{4}{|l|}{ Stroke classification, $n(\%)$} \\
\hline TIA & $17(15.2)$ & $10(12.0)$ & 0.573 \\
\hline Ischemic stroke & $87(77.7)$ & $61(73.5)$ & 0.499 \\
\hline Hemorrhagic stroke & $8(7.1)$ & $7(8.4)$ & 0.738 \\
\hline Stroke mimics & $0(0)$ & $5(6)$ & 0.013 \\
\hline \multicolumn{4}{|l|}{ Stroke etiology (TOAST), ${ }^{\mathrm{b}} n(\%)$} \\
\hline Atherothrombotic & $6(5.8)$ & $9(12.7)$ & 0.109 \\
\hline Small vessel & $21(20.2)$ & $17(23.9)$ & 0.555 \\
\hline Cardioembolic & $33(31.7)^{c}$ & $16(22.5)^{\mathrm{d}}$ & 0.183 \\
\hline Other & $4(3.8)$ & $3(4.2)$ & 0.999 \\
\hline \multicolumn{4}{|l|}{ Undetermined } \\
\hline Two or more possible causes & $4(3.8)$ & $1(1.4)$ & 0.650 \\
\hline Negative workup & $25(24.0)$ & $21(29.6)$ & 0.414 \\
\hline Incomplete evaluation & $11(10.6)$ & $4(5.6)$ & 0.286 \\
\hline
\end{tabular}

Numbers are $n(\%)$, median (IQR) as appropriate. COVID-19, coronavirus disease 2019; IQR, interquartile range; $n$, number; NIHSS, National Institutes of Health Stroke Scale; ASPECTS, Alberta Stroke Program Early Computed Tomography Scale; TIA, transient ischemic attack; TOAST, Trial of Org 10172 in Acute Stroke Treatment; AF, atrial fibrillation. ${ }^{a}$ Only includes patients with middle cerebral artery ischemic stroke $(n=77)$. ${ }^{b}$ Only includes patients with ischemic stroke and TIA $(n=175) .{ }^{c}$ Twenty-five patients had a previous history of AF; in 7 patients a diagnosis of AF or atrial flutter was made during admission, and in 1 patient a left ventricle ejection fraction $\leq 35 \%$ was detected. ${ }^{\mathrm{d}}$ Seven patients had a previous history of AF, 8 patients were diagnosed of AF or atrial flutter during admission, and 1 patient had left ventricular noncompaction cardiomyopathy prior history.

Table 3. Stroke treatment and time course

\begin{tabular}{lccc}
\hline & $\begin{array}{l}\text { Pre-COVID-19 } \\
\text { period group } \\
(n=112)\end{array}$ & $\begin{array}{l}\text { COVID-19 } \\
\text { period group } \\
(n=83)\end{array}$ & $p$ value \\
\hline Stroke treatment, $n(\%)$ & & & 0.186 \\
$\quad$ Intravenous thrombolysis & $15(17.2)$ & $16(26.2)$ & 0.411 \\
Mechanical thrombectomy & $19(21.8)$ & $20(32.8)$ & 0.830 \\
Total patients treated (IVT or MT) & $30(34.5)$ & $304(93-760)$ & 0.104 \\
Time course & $197(91.25-645)$ & $36(43.4)$ & 0.043 \\
Median onset-to-door time, min (IQR) & $65(58)$ & $24(16.5-44)$ & 0.592 \\
Onset-to-door within 4.5 h, $n$ (\%) & $26.5(12-55.7)$ & $37(25.7-96)$ & 0.403 \\
Median door-to-CT time, min (IQR) & $35(21-60.5)$ & $101.5(70.2-113.5)$ & 0.910 \\
Median door-to-needle time, min (IQR) & $95(69-120)$ & & \\
Median door-to-groin time, min (IQR) & & & \\
\hline
\end{tabular}

Numbers are $n(\%)$, median (IQR) as appropriate. COVID-19, coronavirus disease 2019; IQR, interquartile range; $n$, number; IVT, intravenous thrombolysis; MT, mechanical thrombectomy; CT, computed tomography. 


\section{Discussion}

We present the results of the impact of the COVID-19 pandemic on acute stroke care in our center during the first 2 months of lockdown in Spain. Until May 14, 2020, there were a total of 236,611 notified COVID-19 cases in Spain, from which 64,408 were from the Madrid region, meaning more than one third of all national cases [6]. On July 17,2020 , there were a total of 73,026 notified COVID-19 cases in the region, and only 8,618 new cases since the period of our study ended [1]. Thus, our data show the impact of acute stroke care from the Spanish epicenter of the pandemic during the epidemic first wave peak.

The main finding of this study is a significant reduction in the number of acute strokes and TIAs admitted to our center coinciding with the first 2 months of lockdown in Spain. This finding seems to be mainly related to a reduction in the number of elderly patients admitted.

Some studies have already shown differences in attention to acute stroke compared with previous periods. Most of them highlight an important reduction in the number of acute strokes attended during the epidemic peak [7-11], showing a milder impact in areas with less COVID-19 cases among the population [12]. The fear to be infected with the virus and the public recommendation to consult the ED only if necessary have been proposed as important factors to avoid seeking urgent hospital care [13-15]. Along the same lines, it has been suggested that patients experiencing milder symptoms or TIAs would avoid or delay medical attention $[9,11,15$, 16]. However, the proportions of TIAs admitted to our center during both periods were similar. NIHSS score at arrival was higher in the COVID-19 period group, but there was no significant difference.

The decrease in the number of acute strokes admitted was more marked in older patients. Fear of catching the coronavirus infection could have been especially relevant among elderly people, given the generalized knowledge that increasing age or preexisting conditions mean poorer outcomes of the infection. However, social distancing during the lockdown may also have played an important role. Isolation could have complicated continuous care in these patients and could have delayed their families to notice the appearance of stroke symptoms $[8,10]$.

Data also suggest a delay in acute care during the pandemic $[10,13,16]$. In our center, this delay was due to an increase in the time necessary to reach the ED. Once again, the reasons for this delay may be found in a climate of fear and isolation generated by the pandemic and also in the situation of prehospital emergency services and transpor- tation [14] surpassed by the huge amount of respiratory patients attended during the epidemic peak and having to apply security protocols that may have slowed down the chain of acute stroke care. In-hospital times evaluated did not show an important increase, preserving chances for candidates to receive recanalization treatment once arrived to our center.

Acute stroke care is especially important in those cases candidate to reperfusion therapies. Data from different centers have shown a decrease of patients treated with thrombolysis or mechanical thrombectomy [7, 10, 14], while other centers highlight a similar proportion of patients treated with any reperfusion therapies [12]. Although we found a decrease in the number of patients reaching the ED within therapeutic time window of intravenous $\mathrm{r}$-tPA, there were no statistical differences between the total number of patients treated with thrombolysis during both periods. One reason may explain this finding: since patients attended during the COVID-19 period were younger, they had less comorbidities, meaning a lower presence of contraindications to thrombolysis. We additionally did not find differences between the proportions of patients undergoing mechanical thrombectomy. Thus, our data show the percent of patients treated with reperfusion therapy did not suffer an important fall during the most stressful period of the COVID-19 pandemic.

It is important to highlight that the $12 \%$ of patients who suffered an acute stroke during the COVID-19 period were infected with SARS-CoV-2. Due to the reorganization of the in-hospital pathways, in order to protect other patients and staff, they were transferred to a COVID-19 ward, denying them the benefits offered by a stroke unit. Management of acute stroke patients infected with SARS-CoV-2 has been unequal in different centers $[8,17]$. Once the first hit from the COVID-19 pandemic is over, it may be helpful for the future to dispose of general recommendations from multinational organizations.

Our work has important limitations. First, it is a retrospective study from a single center, lacking functional outcomes or follow-up. Second, due to the climate of uncertainty, there were some changes in the management of acute stroke patients during the analyzed period that may carry heterogeneity of our data. Third, our results show the effects of stroke attention in an area severely hit by the pandemic and may not be representative of other areas with a lower number of COVID-19 cases that were more easily taken on by the healthcare system.

We conclude that during the analyzed period, there was a reduction in the number of acute strokes and TIAs attended in our center, and this drop especially affected 
elderly patients. Despite a delay in their arrival to the ED, in-hospital acute stroke care kept relatively unaffected, which is reflected in similar in-hospital delays and a similar proportion of patients receiving reperfusion therapies compared with the previous year.

We need to be prepared for future outbreaks or new epidemics and preserve acute stroke care, paying special attention to these parts of the chain of care that proved to be more vulnerable during the first wave of the pandemic. Educational campaigns must raise awareness in patients experiencing stroke symptoms, especially in elderly people and their families, highlighting the importance of urgent care in stroke and showing hospitals as safe places. Prehospital services must be reinforced and prepared in case an extreme health care situation occurs again. In our center, some measures helped to avoid in-hospital delays. Specific clean and respiratory pathways were established in the ED and most of stroke patients could be managed avoiding the overcrowded dirty pathway. Maintaining trained staff attending acute stroke was also essential, and urgent neurologic attention was guaranteed through a 24-h shift composed of 2 neurology residents and 1 neurologist.

After adopting tough measures, the Spanish and European epidemic seemed to be controlled, but once these measures have been relaxed, the risk of a new outbreak rises. We believe that sharing our experiences and data may help others to face the enormous challenge of guaranteeing acute stroke attention in difficult conditions. A common international response is mandatory, based on evidence from past experiences.

\section{Statement of Ethics}

This study complies with the guidelines for human studies. It was conducted ethically in accordance with the World Medical Association Declaration of Helsinki. Informed consent was not sought for the present retrospective study; there was no need to interview patients because all data necessary was already recorded as part of the assistance activity; an important number of patients included in the study may have died due to the stroke-related mortality; due to the organization of acute stroke care in the Madrid region, some patients were lost to follow-up in our center. Ethical approval for this study was obtained from the Ethic Committee for Research with medicinal products (CEIm) of the Gregorio Marañón Health Research Institute (IiSGM) (REC number: AIADEA2020).

\section{Conflict of Interest Statement}

The authors declare that there is no conflict of interest.

\section{Funding Sources}

This research received no specific grant from any funding agency in the public, commercial, or not-for-profit sectors.

\section{Author Contributions}

Gabriel Velilla-Alonso, Andrés García-Pastor, and AntonioCarmelo Gil-Núñez conceived the study, researched literature, and made a substantial contribution to the design of the work and to the analysis and interpretation of data. GVA wrote the first draft of the manuscript. AGP and AGN revised critically the article for important intellectual content. Ángela Rodríguez-López, Ana Gómez-Roldós, Antonio Sánchez-Soblechero, Fernando Díaz-Otero, Yolanda Fernández-Bullido, Ana María Iglesias-Mohedano, Pilar Vázquez-Alén, and Marta Vales-Montero were involved in patient recruitment and made a substantial contribution to acquisition and analysis of data. Laura Amaya-Pascasio was involved in protocol development and gaining ethical approval. All authors reviewed and edited the manuscript and approved the final version of the manuscript.

\section{References}

1 Ministerio de Sanidad. Enfermedad por nuevo coronavirus, COVID-19 [Internet]. Madrid: Ministerio de Sanidad, Gobierno de España; $2020 \mathrm{Feb}$. Available from: https://www. mscbs.gob.es/profesionales/saludPublica/ ccayes/alertasActual/nCov-China/documentos/Actualizacion_165_COVID-19.pdf.

2 Rodríguez-Pardo J, Fuentes B, Alonso de Leciñana M, Campollo J, Calleja Castaño P, Carneado Ruiz J, et al. Acute stroke care during the COVID-19 pandemic. Ictus Madrid program recommendations. Neurología. 2020;35(4):258-63.
3 Fuentes B, Alonso de Leciñana M, Calleja-Castaño P, Carneado-Ruiz J, Egido-Herrero J, GilNúñez A, et al. Impacto de la pandemia de COVID-19 en la organización asistencial del ictus. Plan Ictus Madrid. Neurología. 2020;35:363-71.

4 Alonso De Leciñana-Cases M, Gil-Núñez A, Díez-Tejedor E. Relevance of stroke code, stroke unit and stroke networks in organization of acute stroke care: the Madrid acute stroke care program. Cerebrovasc Dis. 2009; 27(Suppl 1):140-7.

5 Corman VM, Landt O, Kaiser M, Molenkamp R, Meijer A, Chu DK, et al. Detection of 2019 novel coronavirus (2019-nCoV) by real-time RT-PCR. Euro Surveill. 2020;25(3):1-8.

6 Instituto de Salud Carlos III [Internet]. Madrid: Ministerio de Ciencia e Innovación, Gobierno de España; 2020 Feb. Available from: https://www.isciii.es/QueHacemos/Servicios/VigilanciaSaludPublicaRENAVE/EnfermedadesTransmisibles/Documents/INFORMES/Informes\%20COVID-19/Infor m e \% 20 n \% c $2 \%$ b a \% 2031 . \% 20 Situaci\%c3\%b3n\%20de\%20COVID-19\%20 en $\% 20$ Espa $\%$ c3\%b1a\%20a\%2014\%20de $\% 20$ mayo\%20de\%202020.pdf. 
7 Pandey AS, Daou BJ, Tsai JP, Zaidi SF, Salahuddin $\mathrm{H}$, Gemmete JJ, et al. Letter: COVID-19 pandemic: the bystander effect on stroke care in Michigan. Neurosurgery. 2020 Jun 4. Available from: https://doi.org/10.1093/ neuros/nyaa252.

8 Rudilosso S, Laredo C, Vera V, Vargas M, Renú A, Llull L, et al. Acute stroke care is at risk in the era of COVID-19: experience at a comprehensive stroke center in Barcelona. Stroke. 2020;51(7):1991-5.

9 Siegler JE, Heslin ME, Thau L, Smith A, Jovin TG. Falling stroke rates during COVID-19 pandemic at a comprehensive stroke center. J Stroke Cerebrovasc Dis. 2020;29(8):104953.

10 Pop R, Quenardelle V, Hasiu A, Mihoc D, Sellal F, Dugay MH, et al. Impact of the Covid-19 outbreak on acute stroke pathways: insights from the alsace region in France. Eur J Neurol. 2020;19:1-5.
11 Naccarato $M$, Scali I, Olivo S, Ajčević $M$, Buoite Stella A, Furlanis G, et al. Has COVID-19 played an unexpected "stroke" on the chain of survival? J Neurol Sci. 2020;414: 116889.

12 Hoyer C, Ebert A, Huttner HB, Puetz V, Kallmünzer B, Barlinn K, et al. Acute stroke in times of the COVID-19 pandemic: a multicenter study. Stroke. 2020;51(7):2224-7.

13 Schirmer CM, Ringer AJ, Arthur AS, Binning MJ, Fox WC, James RF, et al. Delayed presentation of acute ischemic strokes during the COVID-19 crisis. J Neurointerv Surg. 2020; 12(7):639-42.
14 Zhao J, Li H, Kung D, Fisher M, Shen Y, Liu R. Impact of the COVID-19 epidemic on stroke care and potential solutions. Stroke. 2020;51(7):1996-2001.

15 Morelli N, Rota E, Terracciano C, Immovilli P, Spallazzi M, Colombi D, et al. The baffling case of ischemic stroke disappearance from the casualty department in the COVID-19 era. Eur Neurol. 2020;83(2):213-5.

16 Teo KC, Leung WCY, Wong YK, Liu RKC, Chan AHY, Choi OMY, et al. Delays in stroke onset to hospital arrival time during COVID-19. Stroke. 2020;51(7):2228-31.

17 Bersano A, Kraemer M, Touzé E, Weber R, Alamowitch S, Sibon I, et al. Stroke care during the COVID 19 pandemic: experience from three large European countries. Eur J Neurol. 2020;27(9):1794-800. 